Elsevier required licence: (C) $<2015>$. This manuscript version is made available under the CC-BY-NC-ND 4.0 license http://creativecommons.org/licenses/bync$\mathrm{nd} / 4.0 /$ 


\title{
A Fast Affine-invariant Features for Image Stitching under Large Viewpoint Changes
}

\author{
Ma Xiaomin ${ }^{\mathrm{a}, *}$, Liu Ding ${ }^{\mathrm{a}}$, Zhang Jian ${ }^{\mathrm{b}}$, Xin Jing $^{\mathrm{a}}$ \\ ${ }^{a}$ The faculty of Automation and Information Engineering, Xi'an University of Technology, Xi'an 710048, China \\ ${ }^{\mathrm{b}}$ Faculty of Engineering and Information Technology, University of Technology (UTS), Sydney, Australia
}

\begin{abstract}
Image alignment and stitching is a popular application on many smart phones, but it is time consuming and creates a critical bottle neck in the course of implementation. In this paper, a fast and high-quality image stitching method is proposed. First, a series of simulated images is obtained by simulating the latitude and longitude angles of a raw image; second, FAST detector is used to detect the features of all the simulated images and described by FREAK (Fast Retina Key-point) before all the feature information is projected to the raw image; third, Hamming distance is used as a feature similarity metric and all the features are matched directly instead of using the repetitive projection in ASIFT (Affine-SIFT). RANSAC is then used to achieve the optimal affine-transformations, and lastly, a weighted average bending algorithm is used to smooth the intensities of the overlapping regions. The experimental results demonstrate that the proposed image stitching method greatly increases the speed of the image alignment process and produces a satisfactory result.
\end{abstract}

Keywords

Image stitching; Image alignment; Affine-invariant features; ASIFT; FREAK

\section{Introduce}

As the customer's demand of enjoying a panorama increases, image stitching attracts more and more attention on smart phones [1]. A panoramic image has a wide field of view and is constructed from a series of images taking one by one and having overlapping areas. To generate a visually natural panorama, image alignment and image blending [2], are necessary. Image alignment is the premise of image blending and used for finding a transformation to make different images totally correspondent in space domain. Hence, the quality of image alignment directly influences the accuracy and efficiency of image stitching [3]. In general, image alignment can be divided into three main categories: first, transformation domain-based method [4]; second, intensity-based method [5]; third, feature-based method [6]. Among them, the feature-based method mainly uses the distinctive features between images, such as contour、edge、statistic features. It has been widely used since the early days of stereo matching [7] and has more lately received popularity for image stitching applications [8]. However, the images to be stitched are often derived from different views, different time or different sensors, this requires image alignment algorithm has good ability to resist affine transformation. Meanwhile the construction of panorama needs a lot of computational power and memory, but smart phones only have limited resources compared to desktop computers. In this paper, we propose a fast and fully affine-invariant image alignment algorithm called AFREAK (Affine-Freak) to fit the efficient stitching on smart phones.

The contributions in this paper are summarized below: (1) A new image alignment algorithm is proposed to realize the fast feature extraction and feature matching, especially when the image views are too different. The proposed algorithm combines the idea of simulating affine transformation in ASIFT (Affine-SIFT) [9]with the rapidity of FAST[10] detector and FREAK[11], so the time efficiency is compatible with fully affine-invariance in AFREAK. (2) Considering that FREAK is a binary descriptor, Hamming distance is used to instead of Euclidean distance, meanwhile, a new matching strategy is proposed which simplifies the redundancy steps in ASIFT. These

*Corresponding author. E-mail address: xunjiatong@163.com (M.Xiaomin). 
changes further improve the rapidity of AFREAK.

The paper is organized as follows. In Section 2 related image alignment algorithms are compared from two aspects: rapidity and affine-invariance. In Section 3 stitching process based on AFREAK is first systematically introduced, then the contributions are explained in detail. In Section 4 the rapidity of AFREAK and effectiveness of image stitching are verified through three groups of experiments. Finally a conclusion is drawn In Section 5.

\section{Related Works}

Lowe et al.[12]proposed a scale invariant feature transformation (SIFT), the algorithm has good distinctiveness and robustness. It is invariant to the change of the illumination, image noise, rotation, scaling, and small changes of the viewpoint. A family of SIFT-like and SIFT-extension algorithms, including principal components analysis (PCA)-SIFT [13], gradient location-orientation histogram (GLOH) [14], DAISY [15] and speed-up robust feature (SURF) [16] are developed. But all of them don't have affine-invariance and cannot favor the applications when there is a large view changes. Morel et al. improved SIFT and proposed a fully affine-invariant image alignment algorithm (ASIFT). It enhances not only the affine-invariance of SIFT, but also has good robustness for viewpoint changes. However, the time consuming caused by SIFT detector and SIFT descriptor has extremely restricted its applications in some fields which has higher requirements for rapidity. Pang et al.[17]proposed a fully affine invariant SURF(FAIR-SURF)algorithm, it is faster than ASIFT. Despite all of these promising algorithms, the rapid computation with low memory and computation complexity is far from being a solved problem. Therefore, the binary robust independent elementary feature (BRIEF) [18], the oriented fast and rotated brief (ORB) [19], the binary robust invariant scalable key-points [20] (BRISK) and the fast retina key-point (FREAK) are presented. Among them, FREAK is the best one[11], which is inspired by Human Visual System (more exactly retina), and proposed in Conference on Computer Vision and Pattern Recognition (CVPR) [11]. It is more compact while remaining robust to scale, rotation and noise, and faster to compute with lower memory load than SIFT or SURF, for example, the computing speed of FREAK descriptor is 77 times faster than SURF descriptor. FREAK descriptor is formed by comparing the image intensities of pairs of symmetrical receptive fields over circular sampling grid pattern, the pattern is similar to the distribution of retina which has higher density of points near the center and the density of points drop exponentially. Rosten et al. presented features from accelerated segment test (FAST), it is a sort of simple and fast feature detector which has repeatability and the capability of noise immunity, in particular, it is faster than SURF detector. The process of feature detection is as follows: considering a circle with the radius $r=3$ pixels around the feature candidate $\mathrm{P}$ and comparing the intensity of $\mathrm{P}$ with other pixels in the circle. If there exists $n$ pixels which are all brighter than $P$ and $n$ is greater or equal to a threshold $(n=9)$, the feature candidate $P$ is defined as a FAST feature. As we know, either FREAK descriptor or FAST detector alone is not enough to realize image alignment, meanwhile, in order to make image alignment algorithm can be adapted to the applications which require both affine-invariance and rapidity.

We herein propose a new image alignment algorithm called Affine-Freak (AFREAK). It not only inherited the rapidity of FAST detector and FREAK descriptor, but also the fully affine-invariant of ASIFT. The method firstly makes use of the idea of stimulating longitude and latitude to ensure the affine-invariant feature extraction and description; secondly, FAST and FREAK are used for feature extraction and description; then an improved matching strategy is used for features matching which accelerates the rapidity further; finally, AFREAK is applied to image stitching and realize the rapid and seamless image stitching.

\section{The Image Stitching Principle Based on AFREAK}

The key enabling idea in the method is: extracting features and matches through image alignment algorithm from series of images, then computing the optimal transformation between images using a large number of correct matches, finally realizing the stitching of a panoramic image. 


\subsection{Framework}

The proposed image stitching method based on AFREAK algorithm contains two sections: (1) Image alignment(including AFREAK feature extraction and description, coarse matching and precise matching); (2) Image blending (including transformation estimation and weighted average blending). Among them, AFREAK feature extraction and description 、 matching are the two improvements, they can realize the fast and accurate extraction of affine-invariant features even when there is a large change of views.Fig.1 gives an overview of the method.

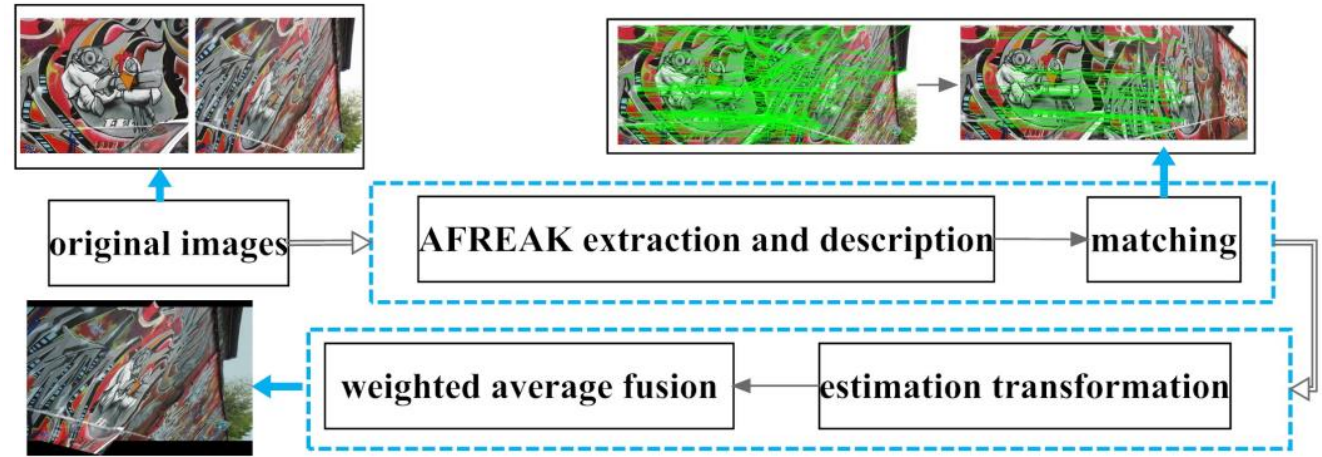

Fig.1. System framework of image stitching

The specific process can be divided into five steps:

(1) Take series of images captured from different viewpoints as reference images and images to be stitched (a pair of image for example in Fig.1);

(2) Quickly extract the affine-invariant features and describe them using AFREAK

(3)Obtain precise matches using Hamming distance [21] and random sample consensus (RANSAC) [22](coarse matches and precise matches are shown in Fig.1 );

(4) Estimate the accuracy affine-transformation $\mathrm{H}$ between the images according to numerous accurate matches, and project the image to be stitched to the coordinates of the reference image using matrix $\mathrm{H}$

(5) Use weighted average algorithm to cope with unsmoothed transition in overlapping region and realize the seamless image stitching (the stitching result is displayed in Fig.1).

\subsection{AFREAK detection and description}

Considering that FAST and FREAK not only have invariance in rotation 、 transformation and brightness, but also are stable in noise and views changes to some degree. Especially, they are faster than SIFT、SURF and BRISK, We proposed a new image alignment algorithm AFREAK. It has fully affine-invariance while keeping the rapidity advantage. If we assume that the camera is located in front of the object, affine transitions of image occur as the camera moves from a frontal view. The tilt between the frontal view and a slanted view is defined as $t$, the camera spin is parameterized by $\psi$ and the zoom is measured by $\lambda$, the angle $\theta$ made by the optical axis with the normal to the image plane is called latitude, the angle $\phi$ caused by the plane containing the normal and the optical axis with a fixed vertical plane is called longitude. In short, the affine transformation is caused by camera motion from a frontal view $\lambda_{0}=1, t_{0}=1, \phi_{0}=\psi_{0}=0$ to an oblique view $\lambda, t, \phi, \psi$, and the affine transformation mainly depends on longitude $\phi$ and latitude $\theta$. $\phi$-rotations of images are realized by tilts with parameter $t=|1 / \cos \theta|[9]$. To digital images, the previous application of an antialiasing filter in the direction of $x$ is needed, i.e., the convolution by a Gaussian with standard deviation $\mathrm{c} \sqrt{t^{2}-1}$, where $\mathrm{c}=0.8$ [12].In AFREAK, the features detection and description are divided into the two steps: $\theta$ and $\phi$ are firstly simulated through the sampling of $t$, then features are detected and described by FAST detector and FREAK descriptor from all stimulated images. The algorithm is described in Fig.2: 


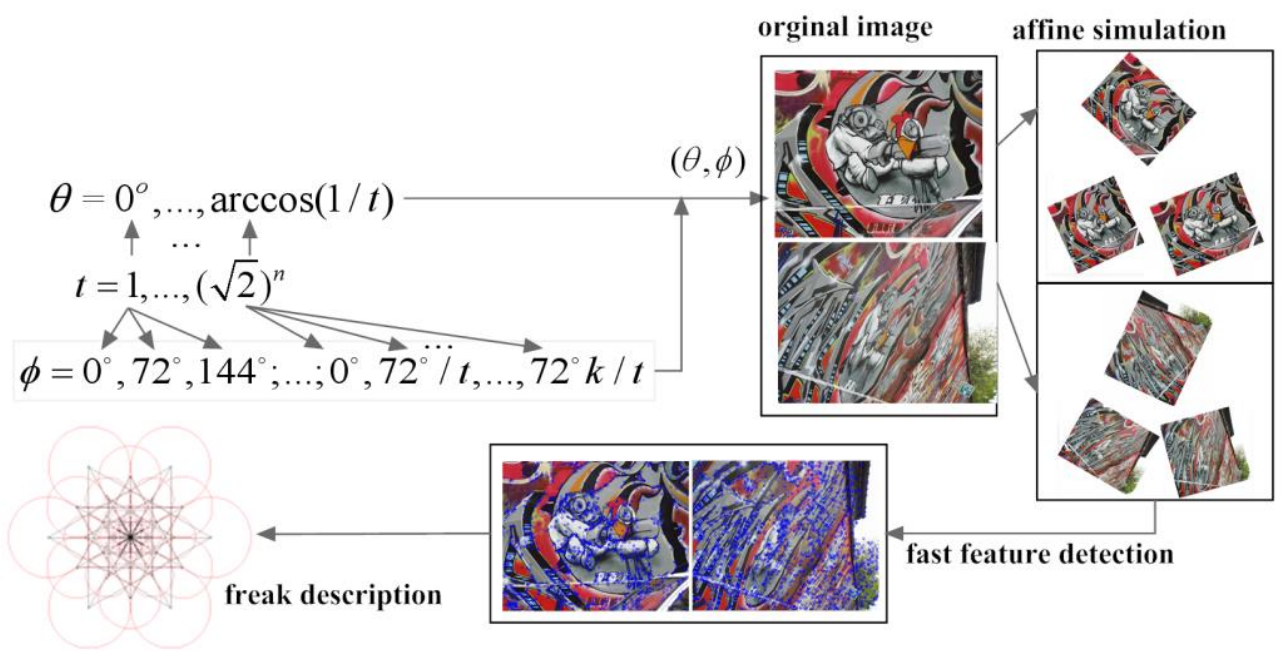

Fig.2. AFREAK extraction

As seen above, firstly computing $\phi$ and $\theta$ based on the parameter $t$, it is a geometric sequence with common ratio $\Delta t=\sqrt{2}$ (The common ratio is experimentally chosen and $\Delta t=\sqrt{2}$ is a good compromise between accuracy and sparsity. Details in [9] ). Latitude angles $\theta$ has a one-to-one correlation to $t$ and $\theta=\arccos (1 / t)$, but longitude angles $\phi$ followed for each $\mathrm{t}$ is one-to-multi and $k$ is the maximal positive integer satisfied $72^{\circ} \mathrm{k} / t<180^{\circ}$; secondly obtaining the stimulated images corresponds to original image using each pairs of $(\theta, \phi)$; then extracting FAST features from all the stimulated images(such as the blue points marked in Fig.2) ; constructing circular sampling grid centered at FAST feature. In FREAK, the pattern has 43 sampling points (shown in Fig.2). For reducing the sensitivity of descriptors to noise and matching the retina model, different kernels size are used in advance for every sample points, i.e., the radius of circle is the standard deviation of Gaussian kernel. The local gradients over pairs of symmetric receptive fields with respect to the center are summed to estimate the orientation of each feature. Let $\mathrm{G}$ be a set of selected pairs used for computing the local gradients, the orientation $O$ can be expressed as:

$$
O=\frac{1}{M} \sum_{P_{o} \in G}\left(I\left(P_{o}^{r_{1}}\right)-I\left(P_{o}^{r_{2}}\right)\right) \frac{P_{o}^{r_{1}}-P_{o}^{r_{2}}}{\left\|P_{o}^{r_{1}}-P_{o}^{r_{2}}\right\|}
$$

Where $\mathrm{M}$ is the count of pairs in $G$ (45 pairs are selected as opposed to few hundreds pairs of other descriptors [11], such as BRISK. This reduces the time complexity of feature extraction.), $P_{o}^{r_{i}}$ is the 2-dimension vector including the spatial coordinates of the center of receptive field, $I\left(P_{o}^{r_{\mathrm{i}}}\right)$ is the intensity of the first receptive field of the pairs $P_{o} .\left\|P_{o}^{r 1}-P_{o}^{r 2}\right\|$ is defined as the Euclidean norm which describes the distance between $P_{o}^{r 1}$ and $P_{o}^{r 2}$. Finally, 43 sampling points are trained to get the most uncorrelated 512 pairs and they are used to construct coarse-to-fine FREAK descriptors. The coarse-to-fine structure is very suitable for saccadic search, i.e., we begin with comparing the first 16 bytes of the FREAK descriptor. If the distance is lower than a given threshold, we make further comparison with the next bytes which represent finer information. Therefore, these cascade comparisons further reduce the time complexity of feature matching.

\subsection{Matching Strategy}

The schematic flows of ASIFT and AFREAK are shown in Fig.3. The whole process of feature detection, description and matching in ASIFT is divided into eight steps and only four steps in AFREAK. 

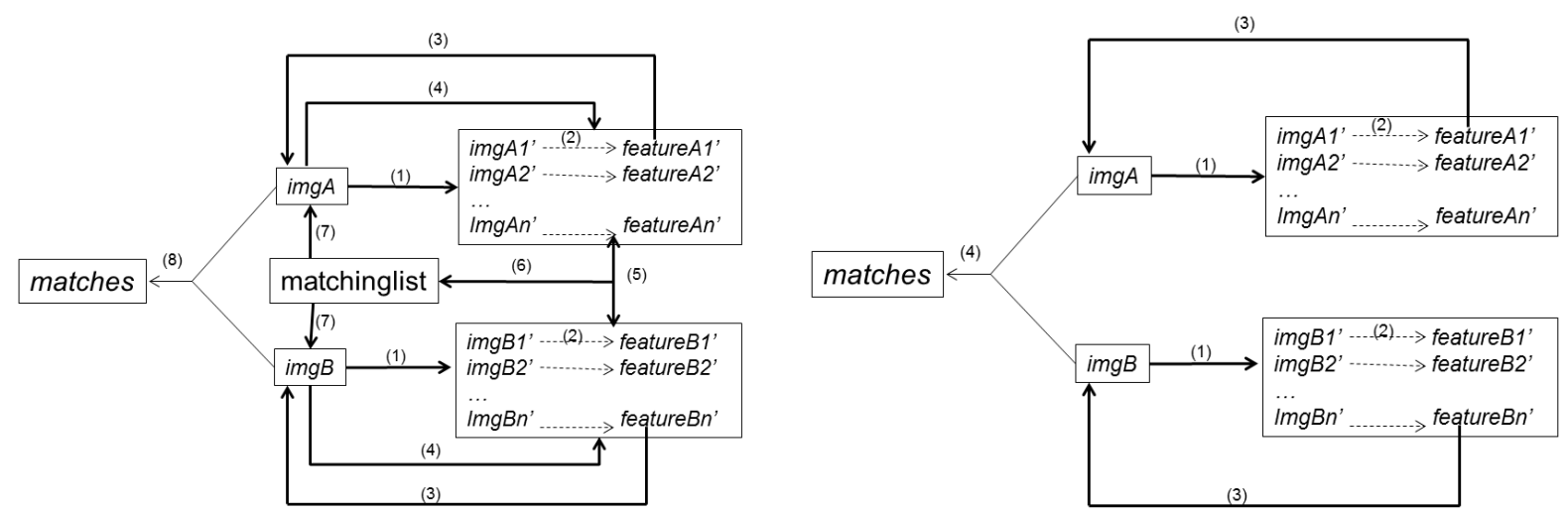

Fig3. ASIFT \& AFREAK. $i m g A$ and $i m g B$ are original image pairs, imgAi and imgBi are stimulated images, featureAi and featureBi' are extracted features of the corresponding stimulated image $(i=1,2, \ldots, m$ and $m$ depends on the number of $(\theta, \phi))$, matchinglist stores matching features of stimulated images, matches are the finally matching features of original images.

In ASIFT, all the imgAi and imgBi are firstly obtained, featureAi and featureBi are then extracted by SIFT through (1) and (2); secondly, featureAi and featureBi are projected to $i m g A$ and $i m g B$ according to each $(\theta, \phi)$, and saved in two dynamic array (recorded as keys 1 and keys 2 ) through (3); then keys 1 and keys 2 are back-projected to the corresponding stimulated images to get matchinglist by Euclidean distance through (4) (6); finally, matchinglist is re-projected to $\operatorname{imgA}$ and $\operatorname{imgB}$ through (7), and get the final matches through (8). However, repetitive projections often cause the problem of huge computation. In AFREAK, the process is simplified and the improvements include: 1 ) the redundancy repetitive projection steps (4) (7) are removed. That is, after obtaining keys1and keys 2 in $\operatorname{img} A$ and $\operatorname{img} B$, they are directly matched to get the final matches. The new strategy dramatically reduces the time complexity of feature matching 2 ) combined with saccadic search proposed in [11], we use Hamming distance (bitwise XOR followed by a bit count) to instead of Euclidean distance for matching descriptors. Hamming distance measures the number of substitutions required to change one descriptor into the other. Therefor the time complexity of feature matching is further reduced.

\subsection{The affine transformation estimation}

For unify the coordinates of image to be stitched and reference image, the affine transformation between images is estimated by the obtained matches. Given $p^{\prime}$ and $p$ is a pair of matching points, the affine transformation $H$ between them is expressed as follows [22]:

$$
p^{\prime}=\left[\begin{array}{l}
x^{\prime} \\
y^{\prime} \\
1
\end{array}\right]=\left[\begin{array}{llc}
h_{0} & h_{1} & h_{2} \\
h_{3} & h_{4} & h_{5} \\
h_{6} & h_{7} & 1
\end{array}\right]\left[\begin{array}{l}
x \\
y \\
1
\end{array}\right]=H p
$$

Where $h_{0} 、 h_{1} 、 h_{3} 、 h_{4}$ represent scale and rotation, $h_{2}$ and $h_{5}$ are the displace of vertical and horizontal, $h_{6}$ and $h_{7}$ are the deformation of vertical and horizontal. In order to gain the eight parameters in H, four pairs of matches are needed at least. However, there are inevitably some false matches, RANSAC algorithm is used to get the exact matches and compute the accurate affine transformation. The process of optimal transformation computation is divided into four steps [23]:

(1) Let $M$ be the set of initial matches obtained by AFREAK, four pairs of matches are randomly selected as the initial set of inliers and used to compute the initial transformation $H^{\prime}$;

(2)Obtain the projection coordinates of outliers by transformation $H^{\prime}$ and compute the error between each projection point and its corresponding point, if the error is less than a given threshold, add this point into the set of 
inliers and define this set of inliers as agree set of the current transformation $H^{\prime}$;

(3)If the number of matches in agree set is more than a certain threshold $\mathrm{T}$, retain this agree set else return (1);

(4)After $\mathrm{N}$ times randomly sampling, re-estimate the accurate transformation $H$ according to the agree set which has the largest numbers of inliers. Then the finally obtained transformation $H$ is the optimal affine transformation.

\section{Image Blending}

The aim of image blending is to smooth intensities of the overlapping regions.

\subsection{Luminance adjustment}

Note that images captured from different viewpoints devices and exposure time are often exit luminance differences, it is needed to adjust luminance for eliminating seams caused by them. Here, we make use of the brightness summation of accurate matching points and the first order linear fitting method. The detailed method is as follows [24]:

(1) Summation of the brightness values. First, given $j$ pairs of matches between image1 (the image to be stitched ) and image2 (the reference image), selecting a $l \times l$ pixel $(l=2)$ neighborhood centred at each matching point; second, summing all the intensities of pixels belonged to each neighbourhood and defining it as $q_{j}$; third, saving $q_{j}$ into the vector $s_{i}=\left[q_{1}, q_{2}, q_{3}, \ldots, q_{j}\right] \quad(i=1,2)$;

(2) The first order linear fitting. Through computing the parameter $s_{c}=[2 \times(l-1)+1]^{2} \times j$, two fitted coefficients a and $\mathrm{b}$ are obtained by the first order linear fitting with the $s_{1} / s_{c}$ as input and $s_{2} / s_{c}$ as output;

(3) Adjusting luminance of each pixel in image 1 and image 2 using the fitted coefficients. The relation between adjusted $m^{\prime}$ and unadjusted $m$ is $m^{\prime}=m \times a+b$;

(4) Because the image stitching in this paper is based on color images, the adjustment should be realized by doing the above three steps in each channel of image.

\subsection{Weighted average blending}

Through the above procedures, image can be simply aligned. But there is still obvious seam that is because the intensities of pixels in overlap region are directly added. Hence, weighted average blending method [24] is adopted to eliminate the stitching seam. Let $p$ be a pixel in the overlap region, its intensity value is computed: value $=k \times$ value $L+(1-k) \times$ value $R$, where valueL is the intensity of $p$ in the left image, valueR is the intensity of $p$ in the right image. $k=d R /(d L+d R), d L$ is the distance between $p$ and the left boundary of overlap region, $d R$ is the distance between $p$ and the right boundary of overlap region.

\section{Experiments}

The following three experiments are designed to demonstrate the strong affine-invariance of AFREAK, the effectiveness and rapidity of the stitching method based on AFREAK when the viewpoint is changed. The experimental environment is Windows XP and the algorithm has been implemented in MATLAB and C++.

\section{Experiment1: Image Alignment Under Different Viewpoints}

This experiment has been carried out on several test images which include standard images and actual images. Some examples of test image sequences are shown in Fig.4.
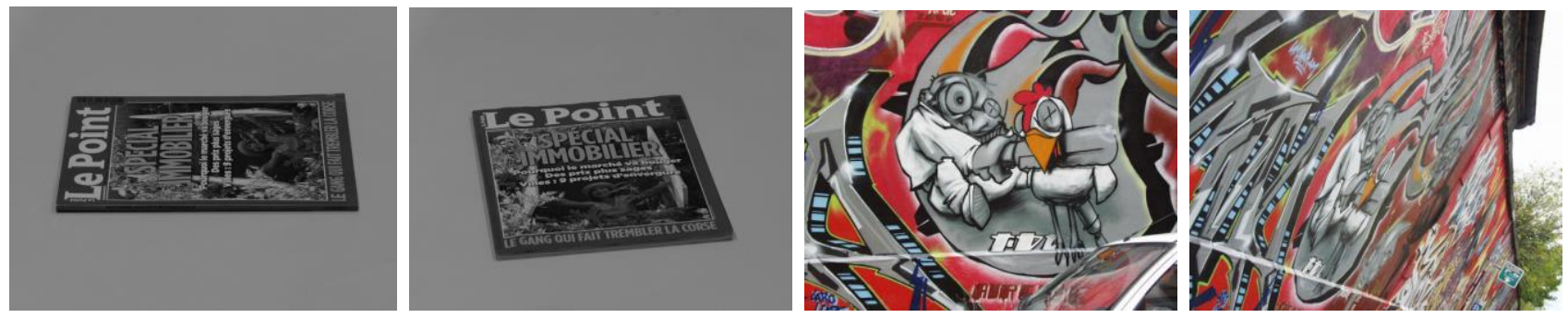

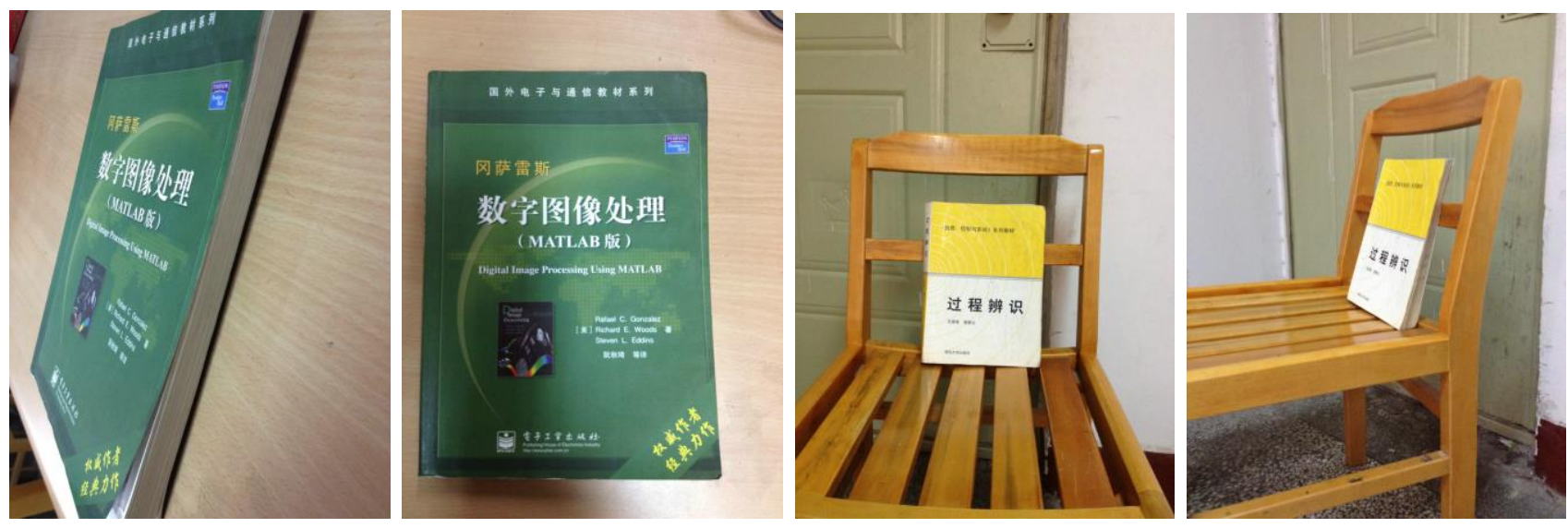

Fig.4. Test images: the upper left two images named magazine and the upper right two images named graffiti are from website http://lear.inrialpes.fr/people/mikolajczyk/. The lower left two images named book-g and the lower right two images named book-y are captured by from actual scene. All the image sequences are with different viewpoints $20^{\circ} 、 40^{\circ} 、 60^{\circ} 、 80^{\circ}$.

The four image sequences are used for feature extraction、 coarse and fine matching under ASIFT and AFREAK to validate the strong affine-invariance of AFREAK (evaluation criterion: total matches and correct matching rate). Fig.5. shows some matching results of the above four sequences.
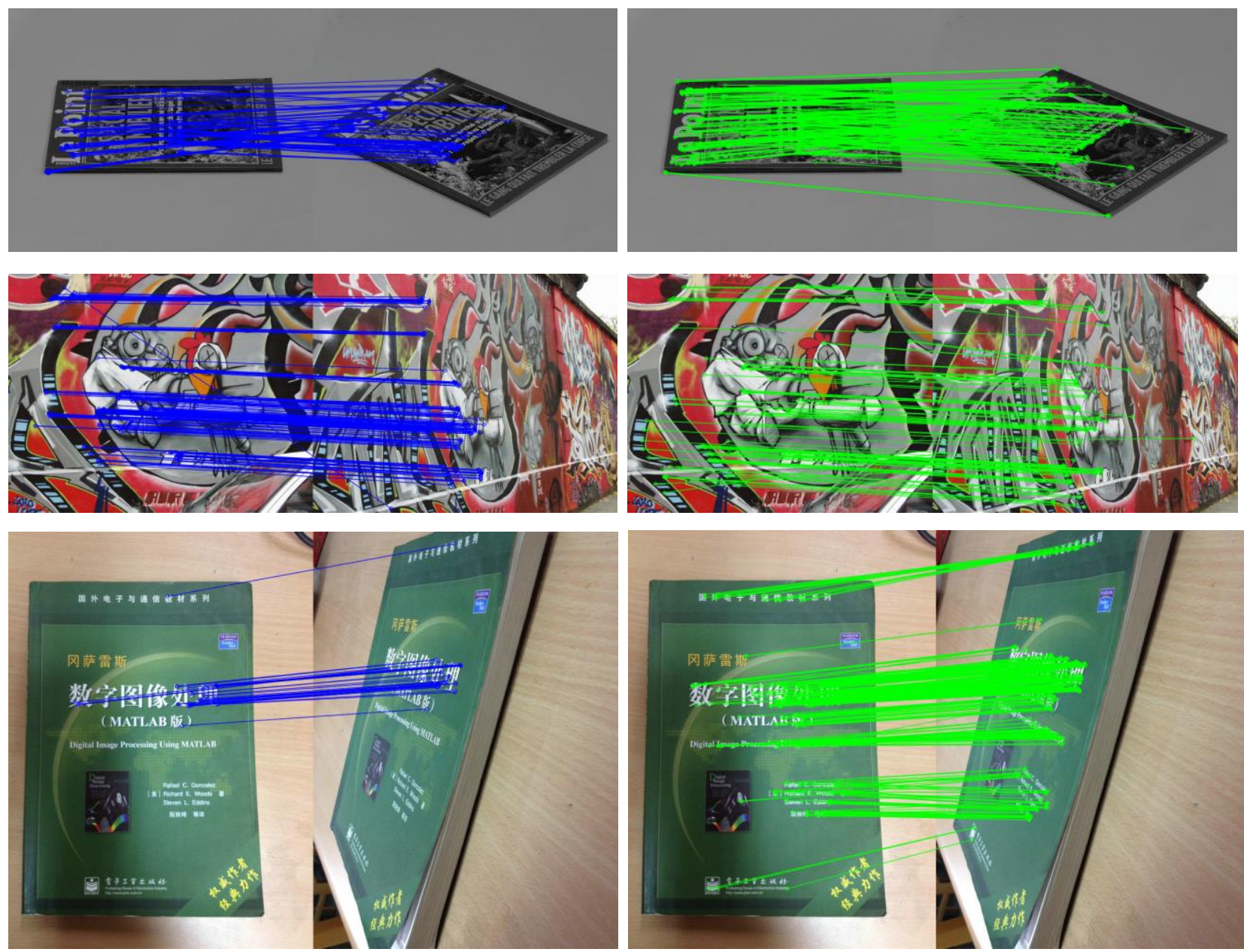

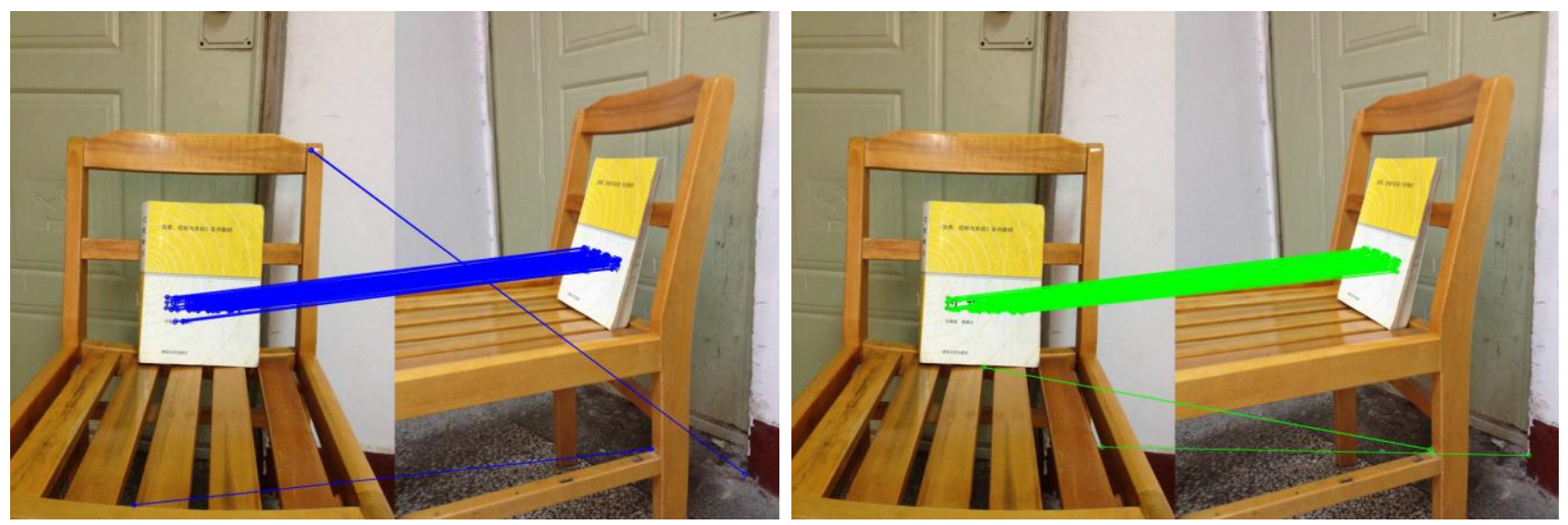

Fig.5. Matching results. Left: ASIFT. Right: AFREAK .The blue and green lines represent the matching line. The first images in each pair of matching results are taken at frontal view, and the second images are taken at $40^{\circ}, 60^{\circ}, 80^{\circ}, 85^{\circ}$

When the viewpoint increases from $40^{\circ}$ to $85^{\circ}$, the two algorithms can both obtain enough correct matches. AFREAK has as strong affine-invariance as ASIFT, which satisfies the requirement of correct matches for computing accurate transformation. In Fig.6, curves of correct matching rate of all the four sequences are shown.
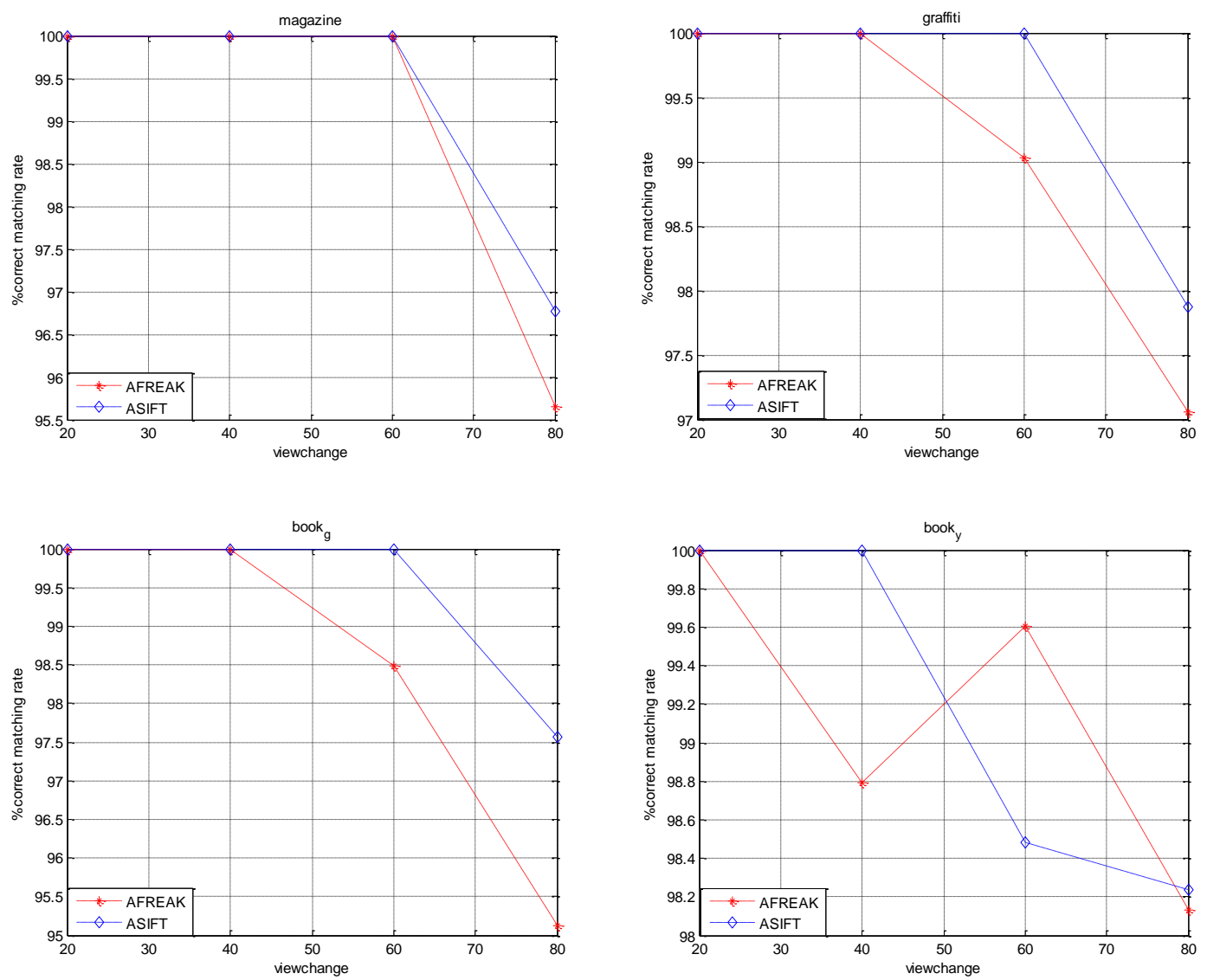

Fig.6. Curves of correct matching rate. The horizontal axis shows the viewpoint changes, and vertical axis shows the percentage of correct matching rate. The curves under AFREAK and ASIFT are separately represented by red "--*--"and blue “-- $\diamond_{--} "$

As the viewpoint increases, the two algorithms both have decreasing trends. However, the correct matching rate 
of AFREAK is larger than $95 \%$ even when the viewpoint is increased to $80^{\circ}$. These comparisons embody the strongly affine-invariance of AFREAK. Table 1 gives the correct matches and total matches.

Table 1

The number of correct and total matches generated from four image sequences with viewpoints changes

\begin{tabular}{|c|c|c|c|c|c|}
\hline \multirow{2}{*}{ test images } & \multirow{2}{*}{ method } & \multicolumn{4}{|c|}{ \#correct matches / \#total matches } \\
\hline & & $20^{\circ}$ & $40^{\circ}$ & $60^{\circ}$ & $80^{\circ}$ \\
\hline \multirow{2}{*}{ graffiti } & ASIFT & $424 / 424$ & $311 / 311$ & $200 / 200$ & $138 / 141$ \\
\hline & AFREAK & $312 / 312$ & $265 / 265$ & $205 / 207$ & $165 / 170$ \\
\hline \multirow{2}{*}{ magazine } & ASIFT & $184 / 184$ & $140 / 140$ & $71 / 71$ & $31 / 31$ \\
\hline & AFREAK & $136 / 136$ & $127 / 127$ & $87 / 87$ & $22 / 23$ \\
\hline \multirow{2}{*}{ book_g } & ASIFT & $504 / 504$ & $365 / 365$ & $75 / 75$ & $40 / 41$ \\
\hline & AFREAK & $327 / 327$ & $159 / 159$ & $65 / 66$ & $39 / 41$ \\
\hline \multirow{2}{*}{ book_y } & ASIFT & $388 / 388$ & $136 / 136$ & $130 / 132$ & $167 / 170$ \\
\hline & AFREAK & $268 / 268$ & $82 / 83$ & $251 / 252$ & $210 / 214$ \\
\hline \multirow{2}{*}{ average correct matching rate } & ASIFT & \multicolumn{4}{|c|}{$99.5 \%$} \\
\hline & AFREAK & \multicolumn{4}{|c|}{$98.8 \%$} \\
\hline
\end{tabular}

From the matching results of the four image sequences with different viewpoints, it can be found that the total matches of ASIFT and AFREAK are both abundant, and the average correct matching rates are close. In summary, AFREAK is effective against the viewpoint changes, the performance provides guarantee for the computation of accurate transformation.

\section{Experiment2:Image Stitching based on AFREAK}

In order to validate the proposed image stitching method, firstly, optimal affine transformations are obtained by AFREAK and RANSAC algorithm; secondly, the coordinates of image to be stitched and reference image are unified by these optimal transformations; finally, seams are eliminated by weighted average blending algorithm. Four groups of stitching results without blending are shown in Fig.7.
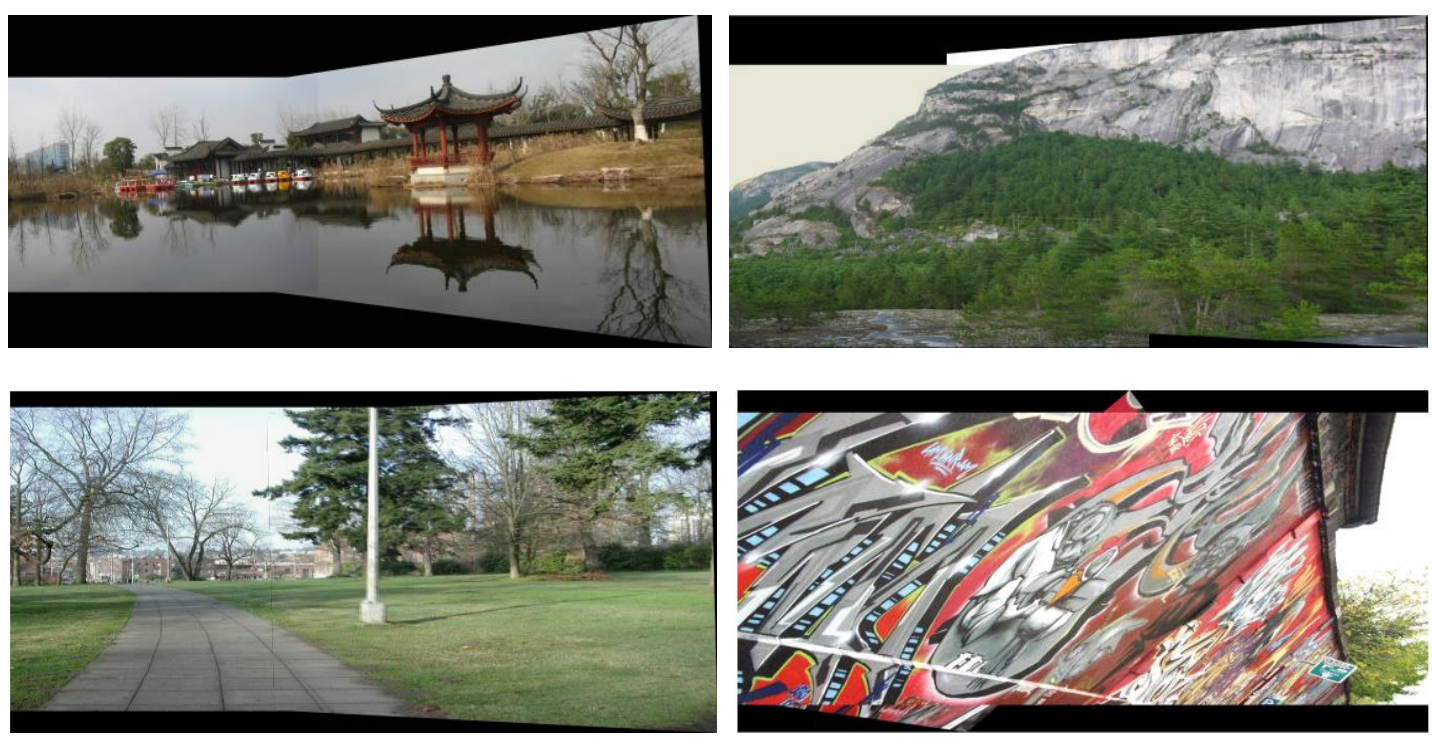

Fig.7. stitched results without blending. It can see that the images are exactly aligned.

But there are still obvious seams, image blending is essential here. The results after image blending are shown in Fig.8. The results demonstrate the effectiveness of the proposed image stitching method. 

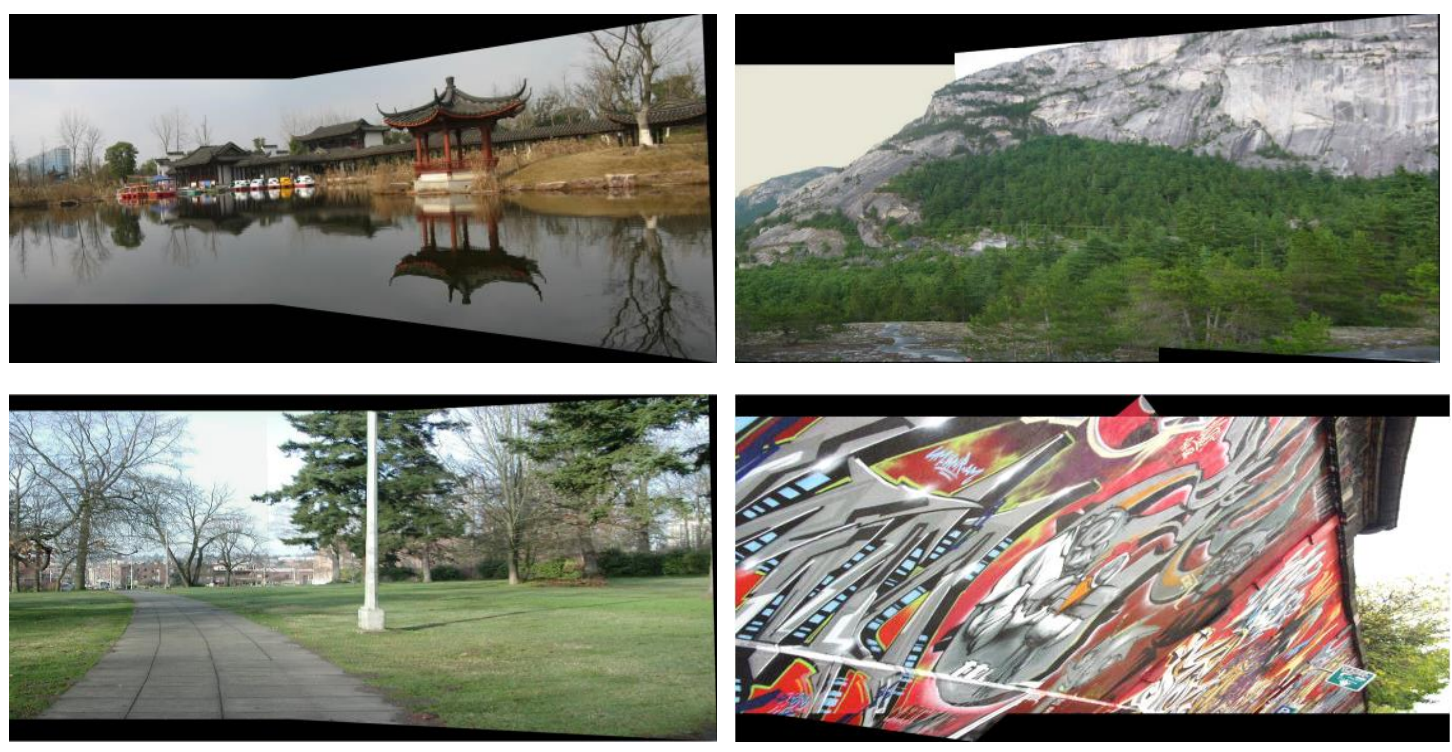

Fig.8. stitched results with blending. It can be seen that the seams are totally eliminated and the images are smoothly stitched.

\section{Experimant3:Running Time Comparison}

To validate quickness of the proposed method, image stitching under AFREAK and ASIFT are carried out on ten groups of images (ASIFT online demo: http://www.ipol.im/pub/demo/my_affine_sift/). The running time of image alignment (including feature extraction and feature matching) and image blending are shown in Table 2.

\section{Table 2}

The running time comparison on ten groups of images (second)

\begin{tabular}{ccccc}
\hline basis & \multicolumn{4}{c}{ Proposed method / Traditional method } \\
\cline { 2 - 5 } & Feature extraction & Feature matching & Image blending & Total time \\
\hline 1 & $12.0 / 24.0$ & $4.0 / 296.5$ & $2.5 / 2.4$ & $18.5 / 322.9$ \\
2 & $11.0 / 30.0$ & $4.0 / 334.5$ & $2.3 / 2.6$ & $17.3 / 367.1$ \\
3 & $11.0 / 20.0$ & $3.0 / 280.4$ & $1.8 / 2.3$ & $15.8 / 302.7$ \\
4 & $12.0 / 19.0$ & $5.0 / 262.7$ & $2.4 / 1.7$ & $19.4 / 283.4$ \\
5 & $13.0 / 28.0$ & $5.0 / 318.0$ & $2.4 / 2.5$ & $20.4 / 348.5$ \\
6 & $11.0 / 25.0$ & $4.0 / 312.2$ & $2.2 / 2.5$ & $17.2 / 339.7$ \\
7 & $13.0 / 21.0$ & $5.0 / 276.5$ & $2.4 / 2.2$ & $20.4 / 299.7$ \\
8 & $12.0 / 42.0$ & $4.0 / 684.6$ & $2.4 / 2.8$ & $18.4 / 729.4$ \\
9 & $14.0 / 48.0$ & $6.0 / 695.6$ & $2.5 / 2.8$ & $22.5 / 746.4$ \\
10 & $16.0 / 47.0$ & $7.0 / 689.2$ & $2.8 / 2.7$ & $25.8 / 738.9$ \\
\hline
\end{tabular}

As the Table2 shows, the feature extraction time and feature matching time of the proposed method have been greatly reduced, this agrees with the qualitative analysis discussed above. To be more specific, the running time of feature extraction and feature matching are separately about 2.4 times and 88.5 times lower than the traditional method. In summary, the proposed method has a better time efficiency. The more experimental results are posted on my blog: http://blog.sina.com.cn/s/blog_12e1f8c520101f7db.html.

\section{Conclusions}

To solve the time-consuming of image stitching under traditional method when the viewpoint change is large, a fast and fully affine-invariant image alignment algorithm AFREAK is presented in this paper. The algorithm is applied to image stitching and has achieved satisfactory stitching effect. First, the idea of simulating longitudes and latitudes are used for reference, FAST features and FREAK descriptors are quickly extracted from all stimulated 
images; then Hamming distance and a new matching strategy are adopted for matching which has further improved the quickness; third, the accurate transformations are obtained by RANSAC algorithm and the seams are eliminated by image blending algorithm. Experimental results indicate the proposed method not only can realize the effective image alignment when the views are changed, but also the time of image alignment and image blending is much less than traditional method.

\section{Acknowledgements}

This work is supported by the National Natural Science Foundation of China under Grant No. 61203345, No.61174101.

\section{References}

[1] Kim S K. A Novel Automatic White Balance for Image Stitching on Mobile Devices. IEEE International Conference on Consumer Electronics (ICCE).(2011)659-660.

[2] Wei L F, Pan L. Optimization method for multi-retinal image mosaic [J].Journal of Image and Graphics.16(12)(2011) 2175-2182.

[3] Yang X H, Wang M. Seamless Image Stitching Method Based on ASIFT [J]. Computer Engineering. 39(2) (2013)241-244.

[4] Tian W G, Guo L. An algorithm for point pattern matching applied to the registration of images with relatively great affine geometric distortion [J].Microelectronics \& Computer. 25(3)(2008)172—174.

[5] Kybic J. High-dimensional mutual information estimation for image registration using control point and intensity [J].IEEE Transaction on Image. 13(8) (2004)1115-1127.

[6] Zitova B, Flusser J. Image registration methods: a survey [J].Image and vision computing. 21(11)(2003)977-1000.

[7] Hannah, M. J. Computer Matching of Areas in Stereo Images. Ph.D. thesis, Stanford University. (1974)

[8] Zoghlami, I., Faugeras, O., and Deriche, R. Using geometric corners to build a 2D stitching from a set of images. In IEEE Computer Society Conference on Computer Vision and Pattern Recognition (CVPR), San Juan, Puerto Rico. (1997) 420-425

[9] Morel J M, Yu G S, ASIFT: a new framework for fully affine invariant image comparison, SIAM J. Imaging Sci. 2 (2) (2009) $438-469$.

[10] Rosten E, Porter R, Drummond T. Faster and better: a machine learning approach to corner detection [J]. IEEE Transactions \& Pattern Analysis and Machine Intelligence. 32(1) (2010)105-119.

[11] Alahi A,Ortiz R, Vandergheynst P. FREAK: Fast Retina Keypoint[C], IEEE Conference on Computer Vision and Pattern Recognition (CVPR). (2012)510-517.

[12] Lowe.D. Distinctive image features from scale-invariant keypoints [J]. International Journal of Computer Vision. 60(2)(2004)91-110.

[13] Y. Ke and R. Sukthankar, PCA-SIFT: A more distinctive representation for local image descriptors, in Proceedings of the IEEE Conference on Computer Vision and Pattern Recognition. 2(2004) 506-513.

[14] K. Mikolajczyk and C. Schmid, A performance evaluation of local descriptors, IEEE Trans. Pattern Anal. Mach. Intell. (2005)1615-1630.

[15] Tola E, Lepetit V, Fua P. Daisy: an efficient dense descriptor applied to wide-baseline stereo. IEEE Transactions on Pattern Analysis and Machine Intelligence. 32(5)(2010)815-830

[16] Bay H, Tuytelaars T. SURF: Speeded up robust features. Computer Vision and Image Understanding (CVIU).110(3) (2008)346-359.

[17] Pang Y W, Li W. Fully affine invariant SURF for image matching [J].Neurocomputing 85(2012)6-10.

[18] M. Calonder, V. Lepetit, C. Strecha, and P. Fua. Brief: Binary robust independent elementary features. Computer Vision-ECCV 2010. (2010)778-792.

[19] E. Rublee, V. Rabaud, K. Konolige, and G. Bradski. Orb: an efficient alternative to sift or surf. 2011.

[20] S. Leutenegger, M. Chli, and R. Siegwart. Brisk: Binary robust invariant scalable keypoints. 2011. 
[21] R.W. Hamming. Error Detecting and Error Correcting Codes[J].The Bell System Technical Journal. 29(2) (1950)147-160.

[22] Fischler, Martin A, Bollers, Robert C. Random Sample Consensus: a paradigm for model fitting with applications to image analysis and automated cartography [J].Communications of the ACM. 24(6) (1981)381-395.

[23] Zhao X Y, Du L M. An Automatic and Robust Image Mosaic Algorithm [J].Journal of Image and Graphics. 9(4)(2004)417-423.

[24] Bu K. Research on Image registration and Mosaic based on SURF [D]. Dalian University of Technology, 2009. 